\title{
A 10-Year Single Centre Experience of Erosive Pustular Dermatosis of the Leg: A Possible Correlation with Atopic Dermatitis?
}

\author{
Ambra Di Altobrando ${ }^{a}$ Annalisa Patrizi ${ }^{a}$ Giulio Vara ${ }^{b}$ Tommaso Bianchi ${ }^{a}$ \\ ${ }^{a}$ Dermatologia - IRCCS Policlinico di S.Orsola, Department of Experimental, Diagnostic and Specialty Medicine, \\ University of Bologna, Bologna, Italy; ${ }^{\text {}}$ Radiology Unit, S.Orsola-Malpighi University Hospital, Bologna, Italy
}

\section{Dear Editor,}

Erosive pustular dermatosis of the leg (EPDL), a rare inflammatory disorder characterized by non-follicular sterile pustules, erosions and crusts, usually occurs on the anterior aspect of the middle third of the leg of elderly patients [1]. The pathogenesis of EPDL is currently unknown and the diagnosis is based upon exclusion of other diseases [1-3].

The frequency of atopic dermatitis (AD) has gradually increased in adults and old people. This increased incidence has been linked to immune system modifications that happen during aging [4-6].

Based on the observation in our clinical practice of a higher incidence of AD in patients with EPDL rather than in the other patients assisted by our Wound Care Unit, we performed a study to evaluate whether these two entities are somehow related.

In the first section, all patients with clinical and histopathological findings consistent with EPDL recorded in our Wound Care Database between January 1, 2010 and September 30, 2020 were included in the study. For every patient we considered gender, age at diagnosis, clinical appearance basing on medical records and clinical pictures collected in our Wound Picture Database. In the second part of the study, we prospectively evaluated our 51 patients for $\mathrm{AD}$. For each patient, the medical data re-

karger@karger.com

(c) 2021 S. Karger AG, Basel

www.karger.com/drm

Karger! corded in the database of the Dermatology Unit of our University Hospital were analyzed, as well as their current clinical picture. For each patient with active $\mathrm{AD}$, the disease severity was calculated by SCORAD.

As regards statistical analysis, data were analyzed by using IBM ${ }^{\circledR}$ SPSS ${ }^{\circledR} 22.0$. According to data distribution, means were compared using the Kruskal Wallis's test, and correlations with the Spearman's test. The Sant'Orsola Hospital institutional review board waived approval and written informed consent for this study.

In total, 51 patients with EPDL were evaluated during the 10 -year study period. The mean patient age was 80.9 \pm 12.4 years, without any clear gender predilection: 24 men (47.1\%) versus 27 women (52.9\%), i.e., a M/F sex ratio of 0.88 . Clinical features included multiple non-follicular pustules of various dimensions, moist erosions and crusts on an erythematous background. Bilateral leg involvement was present in 22 cases (43.1\%), while unilateral involvement was present in 29 cases (56.9\%). The main differential diagnoses (dermatitis cruris pustulosa et atrophicans, eczema and stasis dermatitis, bacterial skin infections, fungal skin infections, traumatic or caustic lesions, localized pretibial bullous pemphigoid, pustular psoriasis, pustular vasculitis, Sneddon-Wilkinson subcorneal pustulosis) were excluded through clinical and histological features, laboratory tests (negative swab 


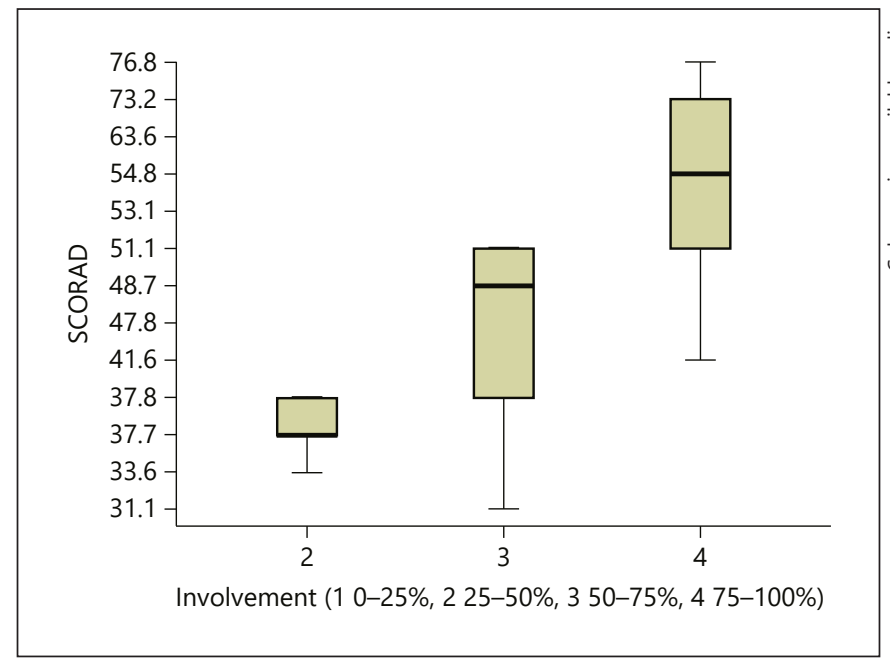

Fig. 1. Correlation between SCORAD and EPDL affected areas. The box plot highlights a significant increase $(p=0.004, r=0.732)$ in percentage of involvement with increasing values of SCORAD. There were 2 patients with an involvement of $25-50 \%$ of the body surface (mean SCORAD $36.367 \pm 2.397$ ), 2 with $50-75 \%$ (mean SCORAD $43.633 \pm 10.920$ ) and 7 with $75-100 \%$ (mean SCORAD $58.700 \pm 13.036)$ (Kruskal-Wallis $p=0.040)$.

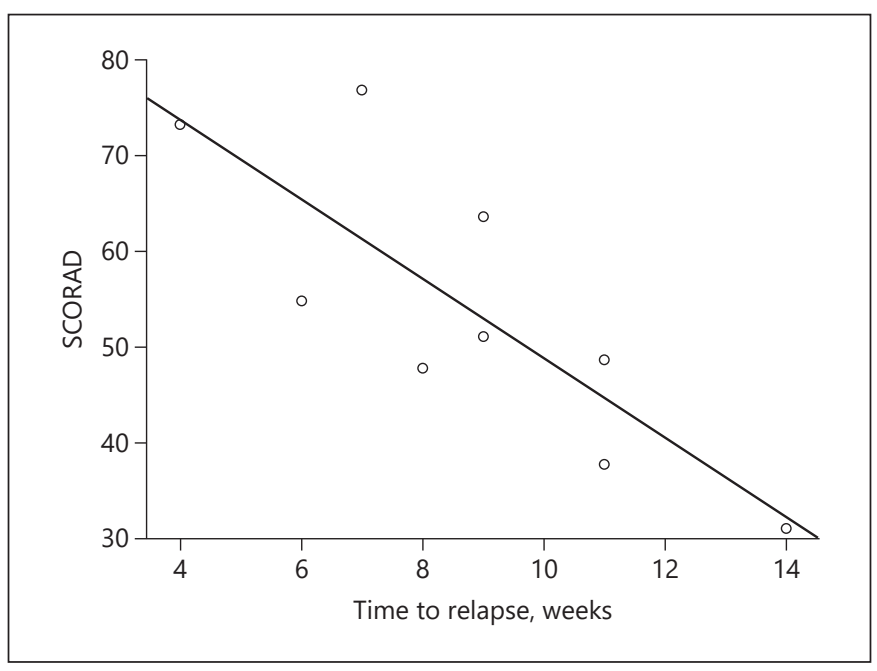

Fig. 2. Correlation between SCORAD and time to relapse. The scatterplot shows a significant correlation $(p=0.015)$ between SCORAD and time to relapse.

for bacteria and fungi) and clinical trend (good response to therapy, albeit sometimes with relapses).

Regarding $\mathrm{AD}, 11 / 51$ patients were not evaluated because of death or inaccessibility, so only $40 / 51$ patients were evaluated for $\mathrm{AD}$. A total of 23 out of them did not have any medical records with a previous diagnosis of $\mathrm{AD}$ and anamnesis was negative for AD. Conversely, a positive previous medical history of AD diagnosed by us on the basis of Hanifin and Rajka's criteria was collected for $17 / 40$ patients with EPDL (42.5\%). Previous episodes of itching had affected $100.0 \%$ (17/17) of patients, periflexural dermatitis $88.2 \%$ (15/17), chronic dermatitis $100.0 \%$ (17/17), while a personal or family history of skin or respiratory allergy $52.9 \%$ (9/17). Among the minor criteria, $11.8 \%(2 / 17)$ of patients had suffered from recurrent conjunctivitis, $58.8 \%(10 / 17)$ from keratosis pilaris, $17.6 \%$ (3/17) from face erythema and $100.0 \%$ (17/17) from dry skin. The high percentage of $\mathrm{AD}$ in EPDL patients resulted remarkable, especially if compared to the other patients assisted by our Wound Care Unit in 2019 (18.7\%). Moreover, the $17 / 40$ patients with an $\mathrm{AD}$ previous diagnosis were assessed for the presence of active $\mathrm{AD}$. As a result, 13/17 patients presented an active $\mathrm{AD}$ according to Hanifin and Rajka's criteria. In particular, itching affected $100.0 \%$ of patients (13/13), periflexural dermatitis $53.8 \%$ (7/13), chronic dermatitis $100.0 \%$ (13/13), while a personal or family history of skin or respiratory allergy $53.8 \%(7 / 13)$. Among the minor criteria, $0.0 \%(0 / 13)$ of patients suffered from recurrent conjunctivitis, $15.4 \%$ $(2 / 13)$ of face erythema, $38.4 \%$ (5/13) of ipopigmented patches, $53.8 \%(7 / 13)$ of keratosis pilaris and $100.0 \%$ $(13 / 13)$ of dry skin; moreover, $38.4 \%$ (5/13) of patients showed Dennie-Morgan fold.

Even if 6/13 patients also presented some positive results after previous PATCH testing, allergic contact dermatitis was excluded. As a matter of fact, the allergens had been carefully avoided and they were not present in the patients' medications or products in use. For the 13 patients with active $\mathrm{AD}$, the disease severity was calculated by SCORAD. A median SCORAD of $48.7 \pm 14.372$ was detected in patients with EPDL and AD. By using the Spearman test, we found that SCORAD directly correlated with the percentage of EPDL affected area $(p=$ $0.004, r=0.732$ ) (shown in Fig. 1) and inversely correlated with time to relapse $(p=0.015, r=-0.773)$. There was a significant difference of SCORAD among patients with different percentage of involvement: there were 2 patients with an involvement of $25-50 \%$ of the body surface (mean SCORAD $36.367 \pm 2.397$ ), 2 with $50-75 \%$ (mean SCORAD $43.633 \pm 10.920$ ) and 7 with $75-100 \%$ (mean SCORAD $58.700 \pm 13.036$ ) (Kruskal-Wallis $p=$ 0.040) (shown in Fig. 2). There was a difference between the SCORAD means of relapsing $(53.878 \pm 15.178)$ and non-relapsing EPDL patients $(41.500 \pm 8.395)$, but it was not statistically significant $(p=0.165)$. 
Our study was limited by the small sample of cases. Nevertheless, the observation of a medical history of AD in $42.5 \%$ of patients with EPDL suggests that EPDL frequently affects patients with AD. Moreover, the direct correlation between SCORAD and EPDL affected areas and the inverse correlation between SCORAD and time to relapse suggest that the severity of EPDL in patients with active $\mathrm{AD}$ may be predicted by the severity of $\mathrm{AD}$ expressed in terms of SCORAD. No statistically significant difference in SCORAD was found between relapsing and non-relapsing patients; still, the observed power of the study was calculated with a post hoc analysis at $46.7 \%$, and a statistically significant result is likely to be found increasing the sample size.

According to our results, attention must be given to medical history of AD in patients suffering from leg lesions such as multiple non-follicular pustules, moist erosions and crusts on an erythematous background. However, further larger studies are needed to confirm our preliminary observations and better define the correlation between these two entities.

\section{Key Message}

The severity of erosive pustular dermatosis of the leg may be predicted by the severity of atopic dermatitis expressed in terms of SCORAD.

\section{Statement of Ethics}

This research complies with the guidelines for human studies and was conducted ethically in accordance with the World Medical Association Declaration of Helsinki. The subjects of this study have given their written informed consent. The paper is exempt from ethical committee approval, since the study was observational and all evaluations related to the study were carried out during normal dermatological control visits.

\section{Conflict of Interest Statement}

The authors have no conflicts of interest to declare.

\section{Funding Sources}

No funding was received.

\section{Author Contributions}

Ambra Di Altobrando: conception and design of the work; acquisition and interpretation of data for the work; drafting of the work; final approval of the version to be published. Annalisa Patrizi: interpretation of data for the work; revising the work critically for important intellectual content; final approval of the version to be published. Giulio Vara: analysis of data for the work; final approval of the version to be published. Tommaso Bianchi: conception and design of the work; revising the work critically for important intellectual content; final approval of the version to be published.

\section{References}

1 Di Altobrando A, Patrizi A, Vara G, Merli Y, Bianchi T. Topical zinc oxide: an effective treatment option for erosive pustular dermatosis of the leg. Br J Dermatol. 2019 Aug.

2 Wantz M, Perceau G, Goeldel AL, Grange F, Bernard P. [Erosive pustular dermatosis of the legs: retrospective study of 16 cases]. Ann Dermatol Venereol. 2011 Feb;138(2):93-9.
3 Nicol P, Perceau G, Barbe C, Bernard P; members of GAD. Erosive pustular dermatosis of the leg: A prospective, multicentre, observational study of 36 cases. Ann Dermatol Venereol. 2017 Oct;144(10):582-8.

4 Bozek A, Fisher A, Filipowska B, Mazur B, Jarzab J. Clinical features and immunological markers of atopic dermatitis in elderly patients. Int Arch Allergy Immunol. 2012; 157(4):372-8.
5 Tanei R, Katsuoka K. Clinical analyses of atopic dermatitis in the aged. J Dermatol. 2008 Sep;35(9):562-9.

6 Ozkaya E. Adult-onset atopic dermatitis. J Am Acad Dermatol. 2005 Apr;52(4):579-82. 